\section{Neue Therapieprinzipien}

\author{
Neue T-Zell-Subpopulationen in der Toleranzentwicklung \\ S. Eyerich \\ ZAUM - Zentrum Allergie und Umwelt, Technische Universität München \\ und Helmholtz Zentrum München
}

T-Zellen nehmen eine zentrale Rolle im adaptiven Immunsystem ein. Sie initiieren Entzündungsprozesse, regulieren Mechanismen der Selbst-Toleranz, induzieren Heilungsprozesse im Gewebe und begründen ein immunologisches Gedächtnis, das ein sofortiges Reagieren auf eine erneute Infektion mit dem gleichen Pathogen erlaubt. T-Zellen stellen daher nicht nur die Homöostase von Barriereorganen, sondern auch die Integrität des gesamten Körpers sicher und erlauben eine kontinuierliche Adaptation an eine sich ändernde Umwelt. T-Zellen werden anhand ihrer Oberflächenmarker unterschieden in $\mathrm{CD}^{+}$- und $\mathrm{CD}^{4+}$-T-Zellen. Die Klassifizierung von $\mathrm{CD} 4^{+}$-T-Zellen wurde begründet durch die Entdeckung von T-Helfer (h) 1 und Th2-Zellen durch Mosmann und Coffman 1986. Intensive Forschung auf dem Gebiet der T-Zellimmunologie führte in den letzten beiden Jahrzehnten jedoch zur Erweiterung dieser T-Helferzellfamilie. Dieser Vortrag gibt einen Überblick über die Vielfalt von $\mathrm{CD}^{+}{ }^{+}$-T-Helferzellen und geht insbesondere auf die Rolle der einzelnen T-Zellsubtypen im Balanceakt zwischen Entzündung und Toleranz ein.

\section{Neue Biologika \\ K. Eyerich \\ Klinik und Poliklinik für Dermatologie und Allergologie, Technische Universität München}

Biologika sind moderne Therapeutika, die in sehr spezifische Signalkaskaden des Körpers eingreifen und deshalb im Optimalfall bei einer Fehlsteuerung mit sehr geringen Nebenwirkungen hohe Wirksamkeit erzielen. In den letzten Jahren wurden über 100 Substanzen für die unterschiedlichsten Erkrankungen in den Bereichen Onkologie, Immunologie und Hämatologie zugelassen. Im Bereich der Allergologie gibt es dagegen bis auf Omalizumab für allergisches Asthma bronchiale bislang keine zugelassenen Biologika. Dieser Vortrag wird einen Überblick über Biologika als solche geben und mögliche Therapieansätze diskutieren, die momentan für das atopische Ekzem, aber auch als Adjuvans in der spezifischen Immuntherapie in der Erprobung sind.

\section{Symposium „Zauberberg 2012“}

\author{
Nahrungsmittelallergie: Update \\ B. K. Ballmer-Weber \\ Allergiestation, Dermatologische Klinik, Universitätsspital Zürich, Schweiz
}

Nahrungsmittelallergien gehören zu den häufigsten Auslösern einer anaphylaktischen Reaktion. Kürzlich publizierte WAO-
Guidelines fassen die Definitionskriterien einer Anaphylaxie zusammen und sollten auch in den klinischen Alltag Eingang finden.

Studien aus Australien belegen eine Zunahme der nahrungsmittelinduzierten Anaphylaxie über zehn Jahre um über $200 \%$. Dies betrifft vor allem die frühkindliche Altersgruppe, wobei Erdnuss als Nahrungsmittel hauptursächlich beteiligt ist. Die höchste Inzidenz für fatale Nahrungsmittelanaphylaxien wurde jedoch im jüngeren Erwachsenenalter festgestellt. Wie neuere Arbeiten beschreiben, legen gerade junge Erwachsene mit Nahrungsmittelallergie ein erhöhtes Risikoverhalten an den Tag, wobei dieses vor allem durch die sozialen Umstände mitbeeinflusst wird.

Nahrungsmittelallergien manifestieren sich klinisch in sehr unterschiedlicher Weise. So können sie sich mit lediglich harmlosen Beschwerden in Form von lokalen oralen Symptomen über Systembeschwerden bis zum anaphylaktischen Schock präsentieren. Faktoren, die zu einem schwereren klinischen Verlauf einer Nahrungsmittelallergie führen können, sind Anstrengung, emotionale Belastung, Alkohol, gleichzeitig eingenommene Medikamente aber auch Komorbiditäten wie Asthma bronchiale oder kardiovaskuläre Erkrankungen. Eine weitere wichtige Einflussgröße bezüglich Schweregrad der klinischen Manifestation ist das Sensibilisierungsmuster gegen die einzelnen Allergene eines Lebensmittels.

Die verdauungsresistenten Allergene wie die Lipid-TransferProteine oder die Speicherproteine führen tendenziell zu schwereren Systemreaktionen als die labilen Bet-v-1-assoziierten Allergene, die eher zu lokalen auf die Mundschleimhaut begrenzten Beschwerden führen. Zunehmend stehen uns im klinischen Alltag solche Allergenkomponenten diagnostisch zur Verfügung. Sie können mithelfen, ein „Risikoprofil“ eines Patienten zu erstellen.

\section{Rehabilitation im Hochgebirge \\ M. Möhrenschlager, G. Menz \\ Hochgebirgsklinik Davos, Schweiz}

Hintergrund: Pneumologische/dermatologische Erkrankungen und Allergien gelten heute als Volkskrankheiten mit einer hohen Belastung für die Betroffenen und das Gesundheitssystem. Zur umfassenden Behandlung gehören neben der kurativen Versorgung rehabilitative Angebote. Für die Rehabilitation im Hochgebirge gibt es eine positive Evidenzlage sowohl für die Behandlung pneumologischer als auch dermatologischer Krankheitsbilder, insbesondere Asthma bronchiale und atopische Dermatitis [Menz 2007, Schultze-Werninghaus 2008]. Um die Evidenzlage zu verbessern, wurde die Davoser-Outcome-Studie (DOS) konzipiert und durchgeführt. Mit der DOS-Studie sollen kurz-, mittel- und langfristige Effekte des Klinikaufenthaltes nachgewiesen werden. Die Studie ist angelegt auf Messzeitpunkte (Aufnahme, Entlassung, 6-, 12-, 24-Monats-Katamnese) und betrachtet somatische, funktionale, psychosoziale, behandlungsbezogene, soziodemografische und gesundheitsökonomische Parameter aus unterschiedlicher Perspektive.

Ergebnisse und Schlussfolgerungen: Die bisher vorliegenden Ergebnisse der DOS-Studie belegen die kurz- und mittelfristigen 